\title{
Phosphorylation of phospholipase D1 and the modulation of its interaction with RhoA by CAMP-dependent protein kinase
}

\author{
Min-Jung Jang ${ }^{1}$, Min-Jung Lee ${ }^{1}$ \\ Hae-Young Park ${ }^{1}$, Yoe-Sik Bae ${ }^{1}$ \\ Do Sik Min ${ }^{2}$, Sung Ho Ryu ${ }^{3}$ \\ and Jong-Young Kwak ${ }^{1,4}$ \\ ${ }^{1}$ Medical Research Center for \\ Cancer Molecular Therapy and \\ Department of Biochemistry \\ College of Medicine, Dong-A University \\ Busan 602-714, Korea \\ ${ }^{2}$ Department of Physiology \\ College of Medicine, The Catholic University of Korea \\ Seoul 137-701, Korea and \\ ${ }^{3}$ Department of Life Science \\ Pohang University of Science and Technology \\ Pohang 790-784, Korea \\ ${ }^{4}$ Corresponding author: Tel, 82-51-240-2928; \\ Fax, 82-51-241-6940; E-mail, jykwak@daunet.donga.ac.kr
}

Accepted 3 March 2004

Abbreviations: dbcAMP, dibutyryl cAMP; dbcGMP, dibutyryl cGMP; PKA, cAMP-dependent protein kinase; PKG, cGMP-dependent protein kinase; PLD, phospholipase D

\begin{abstract}
Agents that elevate cellular CAMP are known to inhibit the activation of phospholipase D (PLD). We investigated whether PLD can be phosphorylated by CAMP-dependent protein kinase (PKA) and PKA-mediated phosphorylation affects the interaction between PLD and RhoA, a membrane regulator of PLD. PLD1, but not PLD2 was found to be phosphorylated in vivo by the treatment of dibutyryl cAMP (dbcAMP) and in vitro by PKA. PKA inhibitor (KT5720) abolished the dbcAMP-induced phosphorylation of PLD1, but dibutyryl cGMP (dbcGMP) failed to phosphorylate PLD1. The association between PLD1 and Val14RhoA in an immunoprecipitation assay was abolished by both dbcAMP and dbcGMP. Moreover, RhoA but not PLD1 was dissociated from the membrane to the cytosolic fraction in dbcAMP-treated cells. These results suggest that both PLD1 and RhoA are phosphorylated by PKA and the interaction between PLD1 and RhoA is inhibited by the phos-
\end{abstract}

phorylation of RhoA rather than by the phosphorylation of PLD1.

Keywords: Phospholipase D; RhoA; cAMP; Protein kinase A; U87 cells

\section{Introduction}

Phospholipase D (PLD) catalyzes the hydrolysis of phosphatidylcholine to generate free choline and phosphatidic acid (PA). PA can be further metabolized by PA phosphohydrolase to form diacylglycerol and by phospholipase $A_{2}$ to form lysophosphatidic acid. Moreover, PA and its metabolites act as second messengers in the regulation of secretion, mitogenesis, and cytoskeletal reorganization (Exton, 1999; Liscovitch et al., 2000)

PLD is tightly regulated by a variety of hormones, growth factors, cytokines, and other agonists (Singer et al., 1997; Frohman et al., 1999). Two types of PLD have been cloned, PLD1 ( 120 kDa) and PLD2 ( 105 $\mathrm{kDa}$ ), which differ both in activation mechanism and subcellular localization (Hammond et al., 1995; Colley et al., 1997). PLD1 is constitutively inactive and is activated by ADP-ribosylation factor (ARF), RhoA, and protein kinase C (PKC) (Hammond et al., 1995). In contrast, PLD2 is constitutively active but insensitive to ARF and RhoA (Colley et al., 1997). Previous studies have demonstrated that the effector region (switch I) of RhoA interacts with the C-terminus of human PLD1 (Bae et al., 1998; Yamazaki et al., 1999; Cai and Exton, 2001). Moreover, in vivo studies have shown that PLD can be activated by the direct binding of RhoA (Du et al., 2000; Xie et al., 2002).

The intracellular accumulation of CAMP leads to functional inhibition in various cells. Increased cAMP in neutrophils lead to the subsequent inhibition of PLD, suggesting that cross-talk is present between the PLD and the cAMP-dependent pathways (Agwu et al., 1991; Tyagi et al., 1991). Our previous study showed that PLD activation was inhibited by CAMPdependent protein kinase (PKA) in a cell-free system of neutrophils (Kwak and Uhlinger, 2000). A component of the plasma membrane has been identified as the target of inhibition by PKA, and the dissociation of the phosphorylated active form of RhoA from the plasma membrane may be involved in the downregulation of PLD activity (Kwak and Uhlinger, 2000). However, no prior evidence of the direct phosphor- 
ylation of PLD by PKA in vivo and in vitro has been presented. Moreover, direct involvement of the phosphorylations of RhoA and/or PLD in the inhibition of their association is not clearly understood although RhoA phosphorylation by PKA was shown to enhance or inhibit the association between RhoA and its binding proteins, such as Rho GDP dissociation inhibitor (RhoGDI) and Rho kinase (Lang et al., 1996; Dong et al., 1998; Forget et al., 2002; Ellerbroek et al., 2003; Qiao et al., 2003).

We report here that PLD1 is a substrate of PKA, and that interaction between PLD1 and RhoA is inhibited by RhoA phosphorylation rather than PLD phosphorylation.

\section{Materials and Methods}

\section{Reagents}

Dibutyryl cAMP (dbcAMP), dibutyryl cGMP (dbcGMP), protein $A$-agarose, and the catalytic subunit of $P K A$ (cPKA) were purchased from Sigma (St. Louis, MO); $\left[\gamma^{32} \mathrm{P}\right] A T P \quad(3,000 \mathrm{Ci} / \mathrm{mmol})$ and $\left[\gamma^{32} \mathrm{P}\right] \mathrm{Pi} \quad(3,000$ $\mathrm{Ci} / \mathrm{mmol}$ ) from NEN Life Science Products (Boston, MA); Dulbecco's modified Eagle's medium (DMEM) and geneticin (G418) from Gibco-BRL (Grand Island, NY); KT5720 and KT5823 from Calbiochem (La Jolla, CA); anti-Myc antibody from Santa Cruz Biotechnology (Santa Cruz, CA); antibody directed against phospho-(Ser/Thr) PKA substrate from Cell Signaling Technology; the enhanced chemiluminescence (ECL) kit from Amersham Bioscience (Beverly, MA); and FuGENE6 transfection reagent from Roche Applied Science (Manheim, Germany). Antibodies against PLD1 and PLD2 were generated as previously described (Kim et al., 2000).

\section{Cell culture and transfection}

U87 human glioma cells were maintained in DMEM medium supplemented with $10 \%$ fetal bovine serum (FBS), $100 \mathrm{U} / \mathrm{ml}$ penicillin, and $100 \mu \mathrm{g} / \mathrm{ml}$ streptomycin at $37^{\circ} \mathrm{C}$ in a humidified atmosphere containing $5 \%$ $\mathrm{CO}_{2}$. pcDNA3.1 containing cDNA for PLD1 or PLD2 were used as expression vectors (Min et al., 2001). U87 cells were transfected either with the empty vector as a control, or with the PLD-expression vector using LipofectAMINE (Gibco-BRL), following the procedure recommended by the manufacturer. Transfected cells were subsequently grown in selection medium containing $500 \mu \mathrm{g} / \mathrm{ml}$ G418. After 3 weeks, antibiotic-resistant clones were isolated and expanded for further analysis under selected conditions. Cells over-expressing PLD1 (U87/PLD1) and PLD2 (U87/PLD2) were co-expressed with the Myc epitope-tagged constitutively active form of RhoA
(Val14RhoA). Briefly, cells $\left(2 \times 10^{6}\right)$ in $60 \mathrm{~mm}$ culture dish were incubated with FuGENE6 transfection reagent $(20 \mu \mathrm{l})$ and empty vector or pcDNA3.1 containing the cDNA of Val14RhoA plasmid $(4 \mu \mathrm{g})$ for $24 \mathrm{~h}$.

\section{Immunoprecipitation and Western blot analysis}

Cells $\left(2 \times 10^{7}\right)$ were treated with or without $300 \mu \mathrm{M}$ of dbcAMP or dbcGMP for $10 \mathrm{~min}$ at $37^{\circ} \mathrm{C}$, washed once with cold phosphate buffered saline (PBS), and resuspended in cold lysis buffer $(20 \mathrm{mM}$ Tris- $\mathrm{HCl}, \mathrm{pH}$ 7.4, $50 \mathrm{mM} \mathrm{NaCl}, 1 \%$ Triton X-100, 1\% deoxycholate, $1 \mu \mathrm{g} / \mathrm{ml}$ leupeptin, $1 \mu \mathrm{g} / \mathrm{ml}$ pepstatin A, $1 \mu \mathrm{g} / \mathrm{ml}$ aprotinin, and $1 \mathrm{mM}$ phenylmethylsulfonyl fluoride). The samples were rotated for $1 \mathrm{~h}$ at $4^{\circ} \mathrm{C}$ and cell lysates were separated from insoluble material by centrifugation at $13,000 \mathrm{~g}$ for $5 \mathrm{~min}$ at $4^{\circ} \mathrm{C}$. The samples were precleared using protein $A$-agarose for $1 \mathrm{~h}$ at $4^{\circ} \mathrm{C}$ and incubated with anti-PLD antibodies for $1 \mathrm{~h}$ at $25^{\circ} \mathrm{C}$. The immune complex obtained was then transferred to $40 \mu \mathrm{l}$ of $1: 1$ slurry of protein A-agarose and mixed for $1 \mathrm{~h}$. The beads were pelleted and washed six times with PBS containing $1 \%$ Triton $X-100$. The immunoprecipitated protein complex was subjected to $10 \%$ sodium dodecyl sulfate-polyacrylamide gel electrophoresis (SDS-PAGE) and transferred to nitrocellulose membranes. Membranes were blocked for $1 \mathrm{~h}$ at $25^{\circ} \mathrm{C}$ with a blocking buffer (25 $\mathrm{mM}$ Tris- $\mathrm{HCl}, 0.15 \mathrm{M} \mathrm{NaCl}$, and $0.2 \%$ Tween 20), incubated with primary antibodies (1:1,000 in dilution) overnight at $4^{\circ} \mathrm{C}$, washed, and incubated for $1 \mathrm{~h}$ with secondary antibodies (1:5,000 in dilution) conjugated to horseradish peroxidase. Signals were detected by chemiluminescence.

\section{${ }^{32} \mathrm{P}$ incorporation into PLD}

The phosphorylation of PLD was determined from the amount of $\left[{ }^{32} \mathrm{P}\right] \mathrm{ATP}$ incorporated into the enzyme after immunoprecipitation with specific PLD antibodies. Cells $\left(5 \times 10^{6}\right.$ cells $\left./ \mathrm{ml}\right)$ were prelabeled with 0.2 $\mathrm{mCi} / \mathrm{ml}\left[{ }^{32} \mathrm{P}\right.$ ]orthophosphate for $90 \mathrm{~min}$ and incubated with or without $300 \mu \mathrm{M}$ of dbcAMP or dbcGMP for $10 \mathrm{~min}$ at $37^{\circ} \mathrm{C}$. Phosphorylation of cell extracts with CPKA in vitro was carried out for $30 \mathrm{~min}$ at $30^{\circ} \mathrm{C}$ in a $100 \mu \mathrm{l}$ final volume of phosphorylation buffer (50 $\mathrm{mM}$ Tris- $\mathrm{HCl}, \mathrm{pH} 7.5,10 \mathrm{mM} \mathrm{MgCl}, 1 \mathrm{mM}$ dithiothreitol, protease inhibitor mixture, $100 \mathrm{U}$ CPKA, 0.1 $\mathrm{mM}$ cold ATP, and $\left.2 \mu \mathrm{Ci}\left[{ }^{32} \mathrm{P}\right] \mathrm{ATP}\right)$. Cell lysates were immunoprecipitated using PLD antibodies. Samples were subjected to SDS-PAGE and followed by autoradiography of dried gel.

\section{Preparation of membranes and cytosolic fraction} dbcAMP-treated or -untreated cells were centrifuged and resuspended at $2 \times 10^{7}$ cells $/ \mathrm{ml}$ in ice-cold rela- 
xation buffer $(50 \mathrm{mM}$ Hepes, $\mathrm{pH} 7.4,100 \mathrm{mM} \mathrm{KCl}$, $5 \mathrm{mM} \mathrm{NaCl}, 1 \mathrm{mM} \mathrm{MgCl}, 0.5 \mathrm{mM} \mathrm{EGTA}$, and protease inhibitor mixtures). Cell suspensions were sonicated for $20 \mathrm{~s}$ and centrifuged for $8 \mathrm{~min}$ at 700 g. The supernatants were ultracentrifuged at 100,000 rpm for $45 \mathrm{~min}$ in a Beckman TL-100 ultracentrifuge using a TLA-100.4 rotor. Membrane pellets were washed once and resuspended in cold relaxation buffer.

\section{Results}

\section{Phosphorylation of PLD1 by PKA}

A possible direct phosphorylation of PLD by PKA was examined in vivo or in vitro. U87/PLD1 and U87/PLD2 cells were established by stably transfecting genes for PLD isozymes into U87 cells and were treated with a cAMP-increasing agent, dbcAMP (Figure 1A). The

A

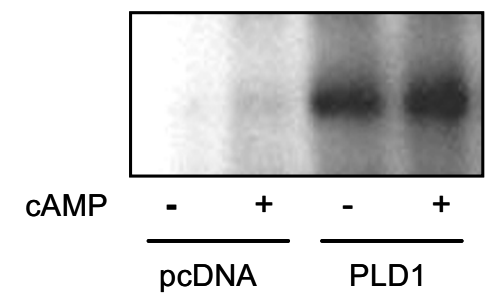

B

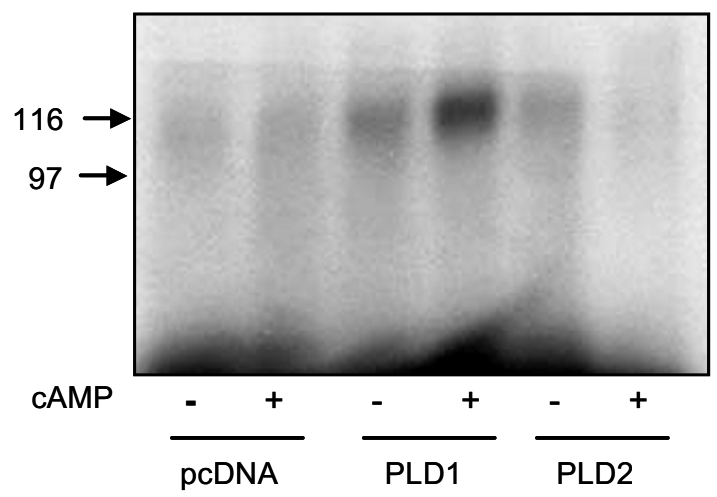

Figure 1. Phosphorylation of PLD1 by cAMP. (A) PLD1 and PLD2 were overexpressed using pcDNA3.1 containing plasmid PLD1 (PLD1) or PLD2 (PLD2) in U87 cells. Cells were transfected with empty vector as a control $(p c D N A)$. Cell lysates $(75 \mu \mathrm{g})$ were immunoblotted with antibodies against PLD1 ( $\alpha$ PLD1) or PLD2 ( $\alpha$ PLD2) as described in "Materials and Methods". (B) After cells were treated with (+cAMP) or without (-cAMP) $300 \mu \mathrm{M}$ dbcAMP for $10 \mathrm{~min}$ at $37^{\circ} \mathrm{C}$, cell lysates $(1 \mathrm{mg} / \mathrm{ml})$ were immunoprecipitated using PLD antibodies $(2 \mu \mathrm{g})$ and blotted with anti-phospho-(Ser/Thr) PKA substrate antibody. Markers indicate molecular weight. Results are representative of three or four experiments. rate of cell proliferation was found not to be significantly different between U87/PLD1 or U87/PLD2 cells and control U87 cells (data not shown). This finding suggests that these stable transfectants may be a suitable model for studying interactions between PLD and its binding proteins, including RhoA. Total cell proteins were obtained from detergent-treated lysates, immunoprecipitated with anti-PLD antibodies, separated by SDS-PAGE, and immunoblotted with the anti-phospho-(Ser/Thr) PKA substrate antibody, which allows detection of typical R-X-X-T/S or R-R-X-T/S consensus sequences for PKA (Schmitt and Stork, 2002). As shown in Figure $1 B$, dbcAMP induced a marked increase in the phosphorylation of PLD1, but not of PLD2. The phosphorylation of PLD was dramatically increased after $10 \mathrm{~min}$ treatment with dbcAMP and this condition was used for further experiments (data not shown).

To determine the effect of dbcAMP on PLD1 phosphorylation by PKA and the specificity of detecting phosphorylated PLD1 with anti-phospho-(Ser/Thr) PKA substrate antibody for PKA-mediated phosphorylation, the effect of a PKA inhibitor was carried out by pretreating cells with the PKA inhibitor, KT5720. The results showed inhibition of PLD1 phosphorylation evidenced by the reduced amount of PLD1 proteins

\section{A}
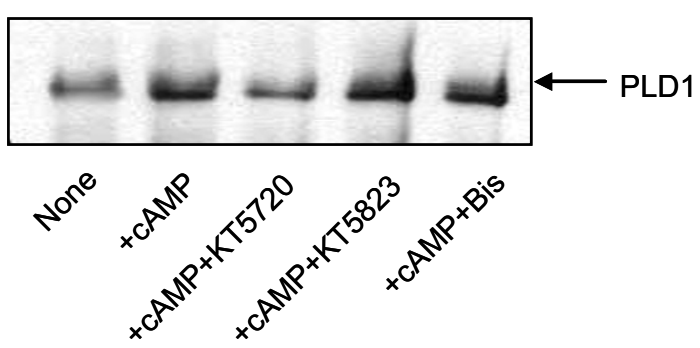

B

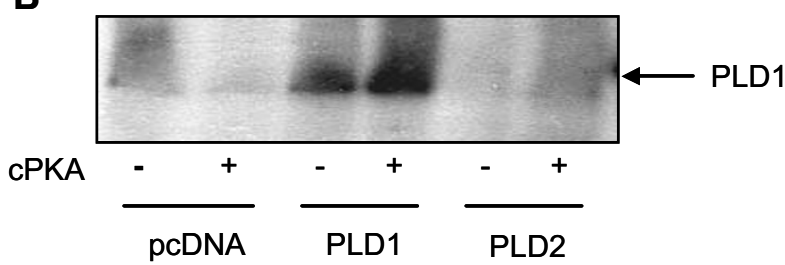

Figure 2. Phosphorylation of PLD1 by PKA. (A) U87 cells overexpressing PLD1 were pre-incubated with or without KT5720 (1 $\mu \mathrm{M})$, KT5823 $(1 \mu \mathrm{M})$, or bisindolylmaleimide I $(10 \mu \mathrm{M})$ (+Bis) for $10 \mathrm{~min}$ and further incubated for $10 \mathrm{~min}$ at $37^{\circ} \mathrm{C}$ in the presence of dbcAMP. (B) Cell extracts were prepared as described in "Materials and Methods" and incubated with (+PKA) or without (-PKA) 100 units of CPKA plus $100 \mu \mathrm{M}$ ATP for $30 \mathrm{~min}$ at $30^{\circ} \mathrm{C}$. Phosphorylation levels were detected as shown in Figure 1B. Results are representative of three independent experiments. 
A

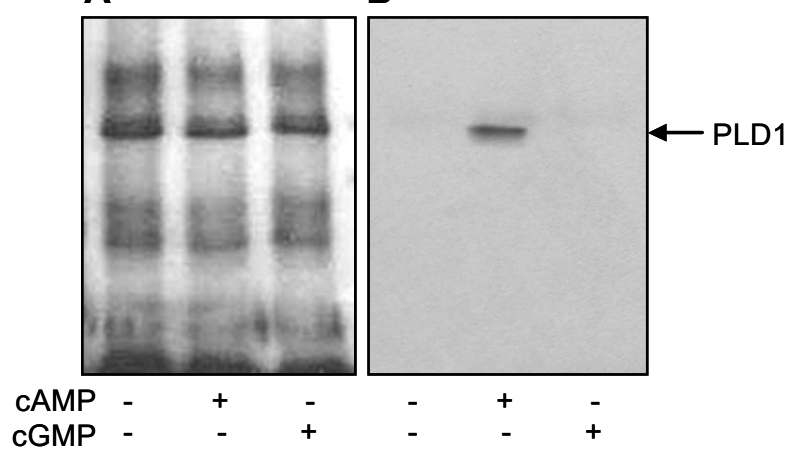

Figure 3. CAMP induced ${ }^{32} \mathrm{P}$ incorporation in PLD1. U87 cells overexpressing PLD1 were labeled with $\left[{ }^{32} \mathrm{P}\right]$ orthophosphate and treated with $300 \mu \mathrm{M}$ of dbcAMP (cAMP) or dbcGMP (cGMP) for $10 \mathrm{~min}$ at $37^{\circ} \mathrm{C}$. Cell lysates were then immunoprecipitated with anti-PLD1 antibody. The immunoprecipitated samples were Western blotted to detect PLD1 (A) and autoradiographed to detect phosphorylated proteins (B). Results are a representative of three experiments.

blotted with anti-phospho-(Ser/Thr) PKA substrate antibody. Whereas, dbcAMP-induced phosphorylation was insensitive to KT5823, an inhibitor of cGMPdependent protein kinase (PKG) and to bisindolylmaleimide I, an inhibitor of PKC (Figure 2A). To assess PKA's ability to phosphorylate PLD in vitro, CPKA plus ATP was added to cell extracts from U87/ PLD1. As shown in Figure 2B, PKA clearly induced the phosphorylation of immunoprecipitated PLD1.

We next examined the effect of cAMP or cGMP on ${ }^{32} \mathrm{P}$ incorporation into PLD, while monitoring total PLD1 proteins immunoprecipitated with anti-PLD1 antibody in parallel by Western blotting (Figure 3). As expected, a phosphorylated band co-localized with the immunodetected band of PLD1. However, the cGMP analogue, dbcGMP had little effect on the phosphorylation of PLD1. Taken together, these results demonstrate that elevated intracellular levels of CAMP phosphorylate PLD1 by activating PKA.

\section{Effect of phosphorylation on the interaction between PLD1 and RhoA}

Binding of the active form of RhoA to PLD was studied by co-immuoprecipitating PLD1 with immobilized RhoA. As shown in Figure 4, the co-transfection of U87/PLD1 cells with Myc epitope-tagged Val14RhoA followed by immunoprecipitation with an antiMyc antibody revealed the co-precipitation of PLD1. However, PLD2 was not detected in the co-immunoprecipitates from Val14RhoA-transfected U87/PLD2 cells (data not shown). This result demonstrates a specific association between PLD1 and the active form of RhoA. To determine whether intracellular
A
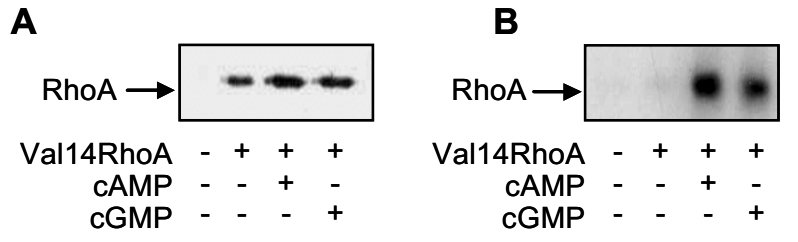

C

D

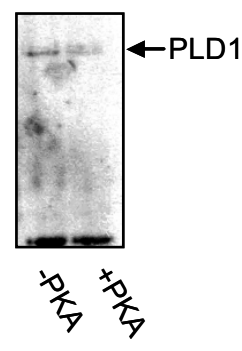

Figure 4. Effect of CAMP and PKA on the interaction between PLD1 and Val14RhoA. A plasmid Myc epitope-tagged Val14RhoA was transiently transfected into U87 cells overexpressing PLD1. Cells $\left(1 \times 10^{7}\right)$ were treated with or without dbcAMP (CAMP) or dbcGMP (cGMP) and cell lysates were immunoprecipitated with antibody against Myc. (A) Expression levels of Val14RhoA were measured by Western blotting using anti-Myc antibody. (B) Cell lysates $(1 \mathrm{mg} / \mathrm{ml})$ were immunoprecipitated using anti-Myc antibody (2 $\mu \mathrm{g})$ and blotted with anti-phospho-(Ser/Thr) PKA substrate antibody. (C) Cells were preincubated with or without $1 \mu \mathrm{M}$ of KT5720 before the addition of dbcAMP (CAMP) or dbcGMP (cGMP). Co-immunoprecipitation of PLD1 was measured by Western blotting using anti-PLD1 antibody. (D) Cell lysates $(1 \mathrm{mg} / \mathrm{ml})$ were treated with (+PKA) or without (-PKA) $100 \mathrm{U}$ of CPKA plus 100 $\mu \mathrm{M}$ ATP as in Figure 2B. Results are a representative of three or four experiments.

CAMP levels regulate the association between PLD1 and the active form of RhoA, RhoA was immunoprecipitated from cells treated with or without dbcAMP and the amount of co-precipitated PLD1 was measured. RhoA was reported to be phosphorylated at Ser188 by both PKA and PKG (Lang et al., 1996; Kwak and Uhlinger, 2000; Sauzeau et al., 2000; Sawada et al., 2001). dbcAMP or dbcGMP induced a marked increase in the phosphorylation of band co-localized with the immunodetected band of Val14RhoA (Figure 4B). The level of PLD1 in Myc immunoprecipitates was markedly reduced in dbcAMP-treated cells in comparison with those in the untreated cells (Figure 4C). The addition of cPKA plus ATP also blocked the association between PLD1 and Val14RhoA in cell extracts (Figure 4D). These results suggest that the dissociation of the PLD1-RhoA complex occurs by the PKA-mediated phosphorylation of PLD1 and/or RhoA. Interestingly, dbcGMP treatment also induced dissociation between PLD1 and RhoA (Figure 4C). Moreover, dbcAMP-induced dissociation was abrogated by the PKA inhibitor, KT5720, whereas dbcGMP- 


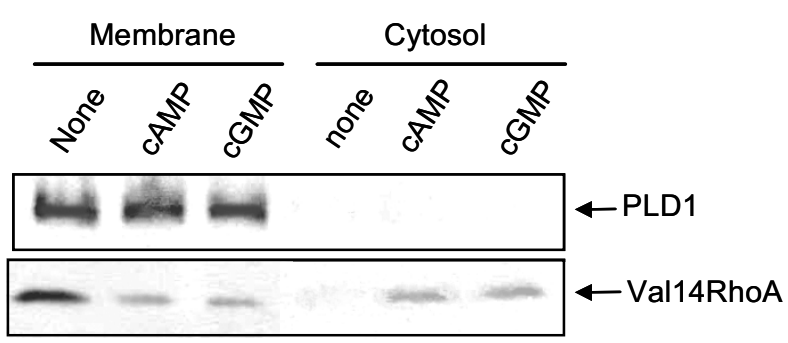

Figure 5. Localization of PLD1 and Val14RhoA in dbcAMP-treated cells. Membrane and cytosolic fractions were prepared from dbcAMPtreated (+CAMP) or untreated cells (-CAMP) as described in "Materials and Methods". Proteins were detected using anti-PLD1 antibody (PLD1) and anti-Myc antibody (Val14RhoA). Results are representative of three independent experiments.

induced dissociation was unaffected by this inhibitor (Figure 4C). Since PLD1 is phosphorylated by only CAMP, as observed in the present study, but RhoA is phosphorylated by both CAMP and CGMP, these results suggest that $\mathrm{RhoA}$ phosphorylation, rather than PLD1 phosphorylation, inhibits the association between PLD1 and RhoA.

\section{Effect of phosphorylation on the localization of PLD1}

Most PLD enzymes are membrane-associated proteins, although cytosolic PLD has been reported in certain cells (Siddiqi et al., 1995; Singer et al., 1997) and the membrane-bound active form of RhoA was extractable by PKA-mediated phosphorylation (Lang et al., 1996; Kwak and Uhlinger, 2000; Forget et al., 2002). We examined whether CAMP-induced phosphorylation could affect the association of PLD with the membrane. PLD1 and Val14RhoA were detected mainly in the membrane fraction of untreated U87/ PLD1 cells. As shown in Figure 5, a large amount of Val14RhoA was detected in the cytosolic fraction after dbcAMP treatment. This observation concurs with previous studies on the dissociation of phosphorylated RhoA from membrane. However, phosphorylated PLD1 was not detected in cytosolic fraction. This result suggests that PLD1 phosphorylated by PKA is not dissociated from membrane although its regulatory factor, RhoA can be easily extracted by PKA-mediated phosphorylation.

\section{Discussion}

The target of phosphorylation in the PKA-mediated inhibition of PLD was shown to be the membrane fraction of neutrophils where the enzyme itself and RhoA were located (Kwak and Uhlinger, 2000). Recent studies indicate that RhoA is phosphorylated by
PKA (Lang et al., 1996; Kwak and Uhlinger, 2000; Ellerbroek et al., 2003). In this study, we investigated whether PLD can be phosphorylated by PKA both in vivo and in vitro. The PLD1 and PLD2 proteins share $50 \%$ homology, and PLD2 does not encode a PLD1like loop region (Colley et al., 1997; Frohman et al., 1999). However, there are several potential PKA phosphorylation sites common to PLD1 and PLD2. Our studies using anti-phospho-(Ser/Thr) PKA substrate antibody or ${ }^{32} \mathrm{P}$ incorporation show that PLD1 but not PLD2 is phosphorylated by PKA. Moreover, these effects were abrogated by PKA inhibitor but were not affected by inhibitors of $P K G$ or $P K C$, indicating that these effects are specific for PKA. In a broader sense, the functional consequence of PLD1 phosphorylation remains to be determined.

Exton et al. suggested that RhoA might activate PLD by direct interaction, since RhoA can activate PLD in vitro (Bae et al., 1998). Although the action of RhoA on PLD1 in vivo has been proposed to take place through indirect pathways (Schmidt et al., 1999), Frohman's group suggested that direct stimulation of PLD1 in vivo by RhoA is critical for significant PLD1 activation (Du et al., 2000). Our studies also revealed that PLD1 associates with the active form of RhoA in vivo. Val14RhoA was shown to suppress basal PLD2 activity in vivo (Xie et al., 2002) but our results indicate that PLD2 cannot bind Val14RhoA. Thus, it is possible that the active form of RhoA may affect other regulatory factor(s) rather than PLD2 itself, since PLD2 activation is independent of RhoA.

The detailed molecular mechanism of the downregulation of PLD by PKA is yet to be clarified. Earlier study suggested that increased intracellular concentrations of CAMP might affect the interaction between the enzyme and its regulators through the phosphorylation of RhoA or the PLD enzyme by PKA (Kwak and Uhlinger, 2000). Here both cAMP-increasing agent and CGMP-increasing agent inhibited the association between PLD1 and Val14RhoA, although the possible physiological role of PKG in PLD signaling is unknown. This result indicates that RhoA phosphorylation by CAMP and CGMP may affect binding of RhoA to PLD1, since PKA and PKG phosphorylate serine188 on RhoA (Lang et al., 1996; Sauzeau et al., 2000; Sawada et al., 2001; Ellerbroek et al., 2003). Moreover, consistent with our findings, PKA-mediated phosphorylation decreased the binding of RhoA to another downstream effector molecule, Rho (Dong et al., 1998). In contrast, the phosphorylation of GTP-bound RhoA by PKA was found to enhance the stabilization of RhoA-RhoGDI (Lang et al., 1996; Ellerbroek et al., 2003; Qiao et al., 2003). Taken together, the PKA-mediated phosphorylation of RhoA may terminate RhoA signaling by enhancing its binding to inhibitory protein, RhoGDI, and reducing its 
binding to its effector molecules, including PLD1. Direct binding and activation of PLD1 by RhoA has been shown to strictly depend on the residue in the switch I region (Bae et al., 1998). Thus, it is possible that the PKA-mediated phosphorylation of Ser188 on RhoA affects a residue responsible for stabilizing the association between RhoA and PLD1. There is another possibility, namely that charge change in RhoA protein caused by the addition of negatively charged Ser188 affects the interaction between PLD and RhoA, since the binding of PLD to RhoA has been shown to involve electrostatic interactions with acidic residues in RhoA (Cai and Exton, 2001). A possibility of RhoA-RhoGDI complex formation promoted by RhoA phosphorylation remains and may interfere with the association between RhoA and PLD1.

PLD1 is localized mainly in the perinuclear membrane, whereas PLD2 is localized in plasma membrane (Colley et al., 1997), and it is known that phosphorylation controls the cellular locations of various proteins. In the present study, treatment of U87/ PLD1 cells with dbcAMP did not affect the localization of PLD1. Our earlier observations showed no change in the level of RhoA in plasma membranes treated with PKA plus ATP, but the addition of cytosol or RhoGDI significantly increased the dissociation of phosphorylated RhoA from a PKA-treated plasma membrane (Kwak and Uhlinger, 2000). In this study, the PLD1 was found in the membrane fraction of dbcAMP-treated cells, whereas membrane-associated RhoA was extractable by dbcAMP. These results suggest that PKA-mediated phosphorylation does not affect the association between PLD1 and the membrane, but that it enhances the dissociation of RhoA from the membrane by promoting its binding with a cytosolic factor. These findings provide further support for our observation that PKA-mediated phosphorylation reduces the interaction between PLD and the active membrane-bound form of RhoA.

Agonist-induced phosphorylation of PLD itself is accompanied by increase in PLD activity, which appears to be mediated by PKC or tyrosine kinase (Exton, 1999; Frohman et al., 1999). Chadhdi et al. has shown that endogenous and expressed PLD1 and PLD2 in RBL-2H3 mast cells are activated by PKA but PLD2 appears to be minimally regulated by PKA (Chahdi et al., 2002). Receptor-stimulated PLD activity but not phorbol ester-stimulated PLD activity was inhibited by PKA (Kwak and Uhlinger, 2000). However, cAMP did not affect unstimulated and phorbol ester-stimulated PLD activities in U87/PLD1 or U87/PLD2 cells (data not shown). In addition, Val14RhoA overexpression alone did not augment the PLD activity of U87/PLD1 cells and cGMP also had no effect on formyl-Met-Leu-Phe-stimulated PLD activity in human neutrophil and the activity in U87/PLD1 cells (unpublished data). Inhibition or down-regulation of PKC does not affect the pervanadate-induced PLD activation in Rat2 cells (Kim et al., 2003). Such effects may be due to differential activation and regulation of PLD by different agonists or involvement of other regulatory factors in the activation of PLD in the cells. The effect of phosphorylation by PKA on the activation of PLD1 is now under investigation.

The present study shows that PLD1 is the substrate for PKA, and PKA-mediated phosphorylation inhibits the interaction between PLD1 and its regulatory factor. Further work is needed to identify residue(s) of PLD phosphorylated by PKA and effect of PLD phosphorylation on the association between itself and other regulatory factors or binding proteins.

\section{Acknowledgement}

This study was supported by the grant R02-200100470 of Korea Science and Engineering Foundation (KOSEF) and by the KOSEF through the Medical Research Center for Cancer Molecular Therapy at Dong-A University.

\section{References}

Agwu DE, McCall CE, McPhail LC. Regulation of phospholipase $\mathrm{D}$-induced hydrolysis of choline-containing phosphoglycerides by cyclic AMP in human neutrophils. J Immunol 1991;146:3895-903

Bae CD, Min DS, Fleming IN, Exton JH. Determination of interaction sites on the small $G$ protein RhoA for phospholipase D. J Biol Chem 1998;273:11596-604

Cai S, Exton JH. Determination of interaction sites of phospholipase D1 for RhoA. Biochem J 2001;355:779-85

Chahdi A, Choi WS, Kim YM, Fraundorfer PF, Beaven MA. Serine/threonine protein kinases synergistically regulate phospholipase D1 and 2 and secretion in RBL-2H3 mast cells. Mol Immunol 2002;38:1269-76

Colley WC, Sung TC, Roll R, Jenco J, Hammond SM, Altshuller $Y$, Bar-Sagi D, Morris AJ, Frohman MA. Phospholipase D2, a distinct phospholipase $D$ isoform with novel regulatory properties that provokes cytoskeletal reorganization. Curr Biol 1997;7:191-201

Dong JM, Leung T, Manser E, Lim L. cAMP-induced morphological changes are counteracted by the activated RhoA small GTPase and the Rho kinase ROKa. J Biol Chem 1998;273:22554-62

Du G, Altshuller YM, Kim Y, Han JM, Ryu SH, Morris AJ, Frohman MA. Dual requirement for rho and protein kinase $C$ in direct activation of phospholipase D1 through G protein-coupled receptor signaling. Mol Biol Cell 2000;11:435968

Ellerbroek SM, Wennerberg K, Burridge K. Serine phosphorylation negatively regulates RhoA in vivo. J Biol Chem 2003;278:19023-31 
Exton JH. Regulation of phospholipase D. Biochim Biophys Acta 1999;1439:121-33

Forget MA, Desrosiers RR, Gingras D, Beliveau R. Phosphorylation states of $\mathrm{Cdc} 42$ and $\mathrm{RhoA}$ regulate their interactions with Rho GDP dissociation inhibitor and their extraction from biological membranes. Biochem J 2002;361: 243-54

Frohman MA, Sung TC, Morris AJ. Mammalian phospholipase $D$ structure and regulation. Biochim Biophys Acta 1999;1439:175-86

Hammond SM, Altshuller YM, Sung TC, Rudge SA, Rose $\mathrm{K}$, Engebrecht J, Morris AJ, Frohman MA. Human ADPribosylation factor-activated phosphatidylcholine-specific phospholipase $D$ defines a new and highly conserved gene family. J Biol Chem 1995;270:29640-3

Kim YR, Cha HY, Lim K, Hwang BD, Hoe KL, Namgung $U$, Park SK. Activation of epidermal growth factor receptor is responsible for pervanadate-induced phospholipase $D$ activation. Exp Mol Med 2003;35:118-24

Kim WJ, Lee MJ, Park MA, Jung JS, Uhlinger DJ, Kwak JY. Dexamethasone enhances phospholipase $D$ activity in M-1 cells. Exp Mol Med 2000;32:170-7

Kwak JY, Uhlinger DJ. Downregulation of phospholipase D by protein kinase $A$ in a cell-free system of human neutrophils. Biochem Biophys Res Commun 2000;267:305-10

Lang $P$, Gesbert F, Delespine-Carmagnat M, Stancou R, Pouchelet M, Bertoglio J. Protein kinase A phosphorylation of RhoA mediates the morphological and functional effects of cyclic AMP in cytotoxic lymphocytes. EMBO J 1996;15: 510-9

Liscovitch M, Czarny M, Fiucci G, Tang X. Phospholipase $D$ : molecular and cell biology of a novel gene family. Biochem J 2000;345:401-15

Min DS, Kwon TK, Park WS, Chang JS, Park SK, Ahn BH, Ryoo ZY, Lee YH, Lee YS, Rhie DJ, Yoon SH, Hahn SJ, Kim MS, Jo YH. Neoplastic transformation and tumorigenesis associated with overexpression of phospholipase $D$ isozymes in cultured murine fibroblasts. Carcinogenesis 2001;22:16417
Qiao J, Huang F, Lum H. PKA inhibits RhoA activation: a protection mechanism against endothelial barrier dysfunction. Am J Physiol Lung Cell Mol Physiol 2003;284:L972-80

Sauzeau V, Le Jeune $H$, Cario-Toumaniantz C, Smolenski A, Lohmann SM, Bertoglio J, Chardin P, Pacaud P, Loirand G. Cyclic GMP-dependent protein kinase signaling pathway inhibits RhoA-induced $\mathrm{Ca}^{2+}$ sensitization of contraction in vascular smooth muscle. J Biol Chem 2000;275:21722-9

Sawada N, Itoh H, Yamashita J, Doi K, Inoue M, Masatsugu $\mathrm{K}$, Fukunaga $\mathrm{Y}$, Sakaguchi S, Sone M, Yamahara Ki K, Yurugi T, Nakao K. cGMP-dependent protein kinase phosphorylates and inactivates RhoA. Biochem Biophys Res Commun 2001;280:798-805

Schmitt JM, Stork PJ. PKA phosphorylation of Src mediates cAMP's inhibition of cell growth via Rap1. Mol Cell 2002;9: 85-94

Schmidt M, Voss M, Weernink PA, Wetzel J, Amano M, Kaibuchi K, Jakobs KH. A role for rho-kinase in rhocontrolled phospholipase $D$ stimulation by the $\mathrm{m} 3$ muscarinic acetylcholine receptor. J Biol Chem 1999;274:14648-54

Siddiqi AR, Smith JL, Ross AH, Qiu RG, Symons M, Exton $\mathrm{JH}$. Regulation of phospholipase D in HL60 cells. Evidence for a cytosolic phospholipase D. J Biol Chem 1995;270: 8466-73

Singer WD, Brown HA, Sternweis PC. Regulation of eukaryotic phosphatidylinositol-specific phospholipase $C$ and phospholipase D. Annu Rev Biochem 1997;66:475-509

Tyagi SR, Olson SC, Burnham DN, Lambeth JD. Cyclic AMP-elevating agents block chemoattractant activation of diradylglycerol generation by inhibiting phospholipase D activation. J Biol Chem 1991;266:3498-504

Xie Z, Ho WT, Spellman R, Cai S, Exton JH. Mechanisms of regulation of phospholipase $\mathrm{D} 1$ and $\mathrm{D} 2$ by the heterotrimeric G proteins $\mathrm{G} 13$ and $\mathrm{Gq}$. J Biol Chem 2002;277: 11979-86

Yamazaki M, Zhang $\mathrm{Y}$, Watanabe H, Yokozeki T, Ohno S, Kaibuchi K, Shibata H, Mukai H, Ono $Y$, Frohman MA, Kanaho $Y$. Interaction of the small $G$ protein RhoA with the C terminus of human phospholipase D1. J Biol Chem 1999; $274: 6035-8$ 